\title{
Riluzole prescribing, uptake and treatment discontinuation in people with amyotrophic lateral sclerosis in Scotland
}

\author{
Kiran Jayaprakash ${ }^{1,3} \cdot$ Stella A. Glasmacher ${ }^{1,3}$ (1) $\cdot$ Bernard Pang $^{3}$ - Emily Beswick ${ }^{1,2,3} \cdot$ Arpan R. Mehta $^{1,2,3,4}$. \\ Rachel Dakin $^{1,2,3}$. Judith Newton ${ }^{1,2,3} \cdot$ Siddharthan Chandran ${ }^{1,2,3,4,5}$. Suvankar Pal ${ }^{1,2,3,4}$. the CARE-MND Consortium
}

Received: 30 April 2020 / Revised: 11 May 2020 / Accepted: 13 May 2020 / Published online: 23 May 2020

(C) The Author(s) 2020

\section{Dear Sirs,}

Riluzole is the only globally licensed drug treatment for amyotrophic lateral sclerosis (ALS), a rapidly progressive neurodegenerative condition. Trials and population studies have reported a survival gain of approximately $2-4$ months with treatment [1,2], and a low frequency of adverse effects [3]. The National Institute for Health and Care Excellence (NICE) recommends that clinicians offer riluzole to all people with ALS (pwALS) in the absence of contraindications [4]. However, in practice, prescribing and uptake are likely to be influenced by a number of clinical factors. Current evidence on rate of treatment discontinuation is limited by selection bias, stemming mainly from trials and small observational studies.

We investigated factors influencing riluzole prescription, uptake and discontinuation using data from a large national disease register with $99 \%$ case ascertainment.

Participants were drawn from the Clinical Audit Research and Evaluation of MND (CARE-MND) platform, a prospectively maintained population-based register comprising longitudinal clinical, and research data for all pwALS in

Kiran Jayaprakash and Stella Glasmacher are joint first authors.

Suvankar Pal

suvankar.pal@ed.ac.uk

1 Centre for Clinical Brain Sciences, University of Edinburgh, Chancellor's Building, 49 Little France Crescent, Edinburgh EH16 4SB, UK

2 Euan MacDonald Centre for Motor Neurone Disease Research, University of Edinburgh, Edinburgh, UK

3 Anne Rowling Regenerative Neurology Clinic, Royal Infirmary, Edinburgh, UK

4 Dementia Research Institute, University of Edinburgh, Edinburgh, UK

5 Department of Clinical Neurosciences, NHS Lothian, Edinburgh, UK
Scotland [5]. We extracted clinical characteristics of people with definite, probable or possible ALS [6]. Summary statistics are reported as median with interquartile range (IQR). Data were analysed using multivariable multinomial logistic regression and are reported as odds ratio (OR) with $95 \%$ confidence intervals (CIs). Missing data were handled using multiple imputation $(m=5)$. Data on the presence/absence of cognitive impairment were used to inform imputation of missing Edinburgh Cognitive and Behavioural Screen (ECAS) scores. Analyses were performed in R (3.6.2.).

768 pwALS were identified between January 2015 and April 2020. 468 pwALS (60.9\%) were male, median age at diagnosis was 68 years (IQR 60-75) and median time from onset to diagnosis was 11 months (IQR 7-19). Site of onset was limb (338, 63.3\%), bulbar $(150,28.1 \%)$, mixed (38, $7.1 \%)$ and pure respiratory $(8,1.5 \%)$. The median ALSFRS$\mathrm{R}$ score was 38 (IQR 31-42) and the median ECAS score was 109/136 (IQR 91-115, $n=253$ ).

Of all pwALS, $632(86.5 \%)$ were offered riluzole and $283(38.7 \%)$ did not commence treatment, which was due to patient preference in 223 (78.8\%) cases (Fig. 1). Older age was significantly associated with pwALS not being offered riluzole, and with not starting riluzole. Sex, diagnostic delay,

6 Department of Neurology, NHS Tayside, Dundee, UK

7 Institute of Neurosciences, NHS Greater Glasgow and Clyde, Glasgow, UK

8 Department of Neurology, NHS Grampian, Aberdeen, UK

9 Department of Neurology, NHS Borders, Melrose, UK

10 Department of Neurology, NHS Dumfries and Galloway, Dumfries, UK

11 Department of Neurology, NHS Fife, Kirkcaldy, UK

12 Department of Neurology, NHS Highland, Inverness, UK 
Fig. 1 Patient flow chart

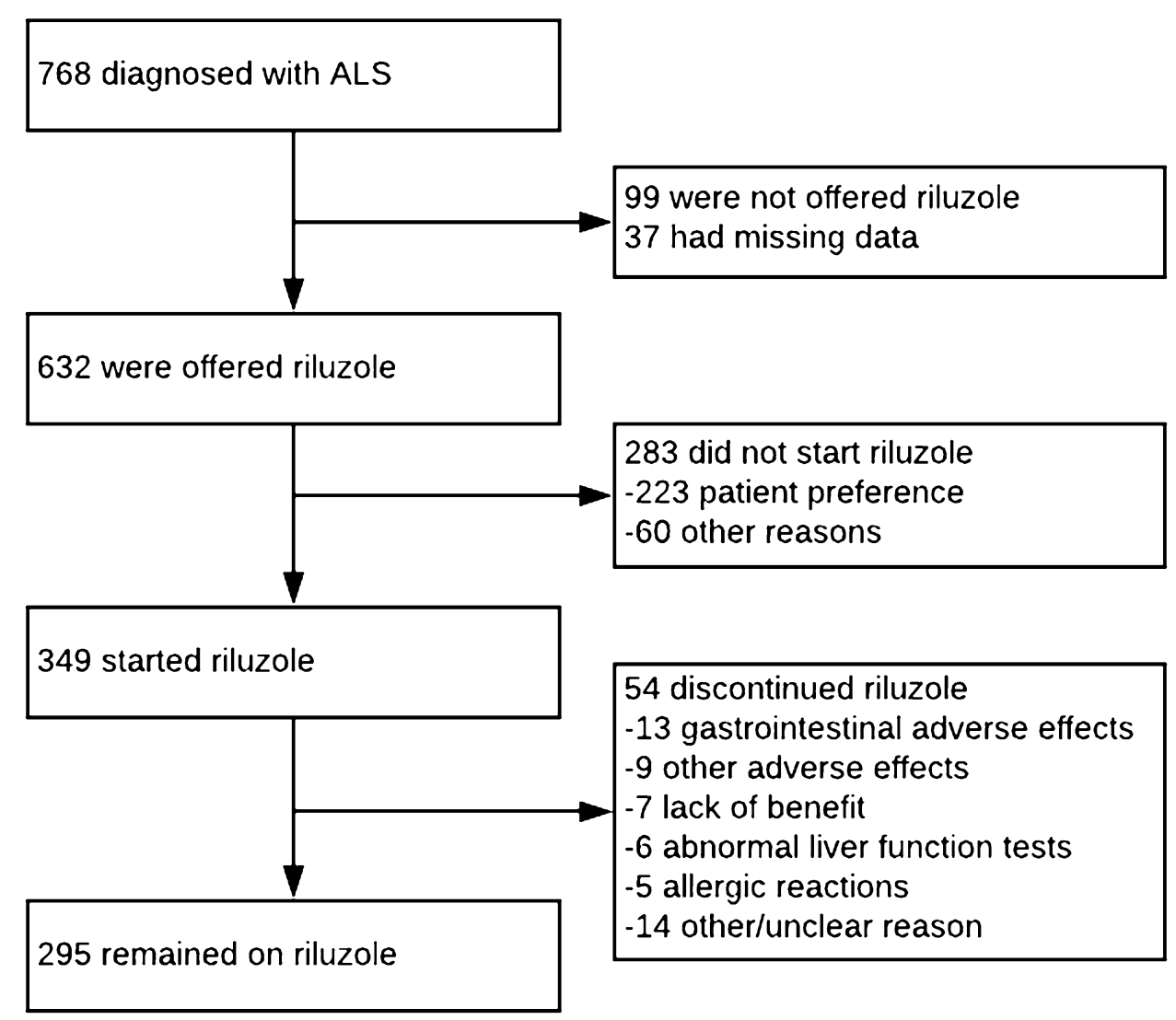

ALSFRS-R total and swallow subscores, and ECAS scores were not associated with prescription or uptake of riluzole (Table 1).

Of those who started riluzole, 54 (15.4\%) subsequently discontinued treatment. The most common reasons for discontinuation were gastrointestinal adverse effects $(24.8 \%)$, including nausea, abdominal discomfort, constipation, and anorexia. Other adverse effects (including fatigue/malaise) accounted for $13.0 \%$, deranged liver function $11.1 \%$, and allergic reactions $9.3 \%$. $13 \%$ discontinued due to ongoing ALS progression. In the remainder, the reason was unclear.
Median time until discontinuation was 3 months for adverse effects and ALS progression (IQR 1.8-3.3 and 2.0-5.0, respectively) and 4.5 months for deranged liver function tests (IQR 2.5-6.0).

The proportion of pwALS in Scotland offered riluzole (86.5\%) is in keeping with previous estimates of $66-100 \%$ in the United Kingdom [7, 8] and 57-85\% internationally $[9,10]$.

Older age was associated with lower rates of riluzole prescription and uptake, which may be because of prescribers' and/or pwALS' concerns about increased vulnerability to

Table 1 Factors associated with being offered riluzole and not starting riluzole, compared to starting riluzole (reference category)

\begin{tabular}{lllc}
\hline Characteristic & $\begin{array}{l}\text { Not offered riluzole }(n=99) \text { OR } \\
(95 \% \text { CIs }) ; p \text { value }\end{array}$ & $\begin{array}{l}\text { Offered but not started riluzole } \\
(n=283) \text { OR }(95 \% \text { CIs })\end{array}$ & $\begin{array}{l}\text { Started riluzole } \\
(n=349)(\text { Reference } \\
\text { category) }\end{array}$ \\
\hline Age at clinical ALS diagnosis (years) & $1.05(1.03,1.08) ; p<0.001$ & $1.03(1.01,1.04) ; p<0.001$ & 1 \\
Male sex & $1.05(0.65,1.69) ; p=0.86$ & $1.07(0.76,1.51) ; p=0.69$ & 1 \\
Time between symptom onset and clinical & $1.01(1.00,1.02) ; p=0.14$ & $1.00(0.99,1.01) ; p=0.61$ & 1 \\
$\quad$ ALS diagnosis (months) & $0.97(0.94,1.01) ; p=0.12$ & $0.99(0.96,1.01) ; p=0.36$ & 1 \\
Total ALSFRS-R score (0-4) & $0.77(0.55,1.06) ; p=0.11$ & $0.87(0.69,1.10) ; p=0.24$ & 1 \\
ALSFRS swallow subscore $(0-48)$ & $0.99(0.97,1.01) ; p=0.21$ & $0.99(0.98,1.00) ; p=0.08$ & 1 \\
ECAS total score (0-136) & &
\end{tabular}

$A L S F R S$-R Amyotrophic lateral sclerosis functional rating scale, $C I$ confidence interval, ECAS Edinburgh Cognitive and Behavioural Screen, OR odds ratio 
adverse effects owing to comorbidity and polypharmacy. Additionally, therapeutic nihilism regarding the modest survival gain conferred by riluzole may be more prominent in this group; however, recent research found that the survival benefit of riluzole is greater in older pwALS [11]. This study, together with our data, emphasises the importance of offering Riluzole to all pwALS.

$15 \%$ of pwMND discontinued riluzole; this figure varied between 4-40\% in previous research [3]. Our data offer insight into reasons for discontinuation, which may inform pre-treatment discussion with pwALS.

Acknowledgements A.R.M. is a Lady Edith Wolfson Clinical Fellow and is jointly funded by the Medical Research Council and the Motor Neurone Disease Association (MR/R001162/1). S.C.'s group is funded by the UK Dementia Research Institute partner funders: the Medical Research Council, Alzheimer's Research UK and the Alzheimer's Society.

CARE-MND Consortium: Shuna Colville (Centre for Clinical Brain Sciences, University of Edinburgh, Chancellor's Building, 49 Little France Crescent, Edinburgh, EH16 4SB, UK; Euan MacDonald Centre for Motor Neurone Disease Research, University of Edinburgh, Edinburgh, UK; Anne Rowling Regenerative Neurology Clinic, Royal Infirmary, Edinburgh, UK), Richard Davenport (Dementia Research Institute, University of Edinburgh, Edinburgh, UK; Anne Rowling Regenerative Neurology Clinic, Royal Infirmary, Edinburgh, UK), Ian Morrison (Department of Neurology, NHS Tayside, Dundee, UK), George Gorrie (Institute of Neurosciences, NHS Greater Glasgow and Clyde, Glasgow, UK), Callum Duncan (Department of Neurology, NHS Grampian, Aberdeen, UK), Myles Connor (Department of Neurology, NHS Borders, Melrose, UK), David Simpson (Department of Neurology, NHS Borders, Melrose, UK), Ondrej Dolezal (Department of Neurology, NHS Dumfries and Galloway, Dumfries, UK), Katja Lassak (Department of Neurology, NHS Fife, Kirkcaldy, UK), Antonella Benvenga (Department of Neurology, NHS Fife, Kirkcaldy, UK), Javier Carod Artal (Department of Neurology, NHS Highland, Inverness, UK).

Funding No funding was required for this study.

Data availability Upon request from the authors.

\section{Compliance with ethical standards}

Conflicts of interest The authors declare that they have no competing interests.

Ethical approval Ethics approval for CARE-MND was provided by the Scotland A Research Ethics Committee (Approval: 15/SS/0126).

Informed consent Participants consented to inclusion in the CAREMND register. Consent from patients was obtained at the time of their registration with the CARE-MND register.

Open Access This article is licensed under a Creative Commons Attribution 4.0 International License, which permits use, sharing, adaptation, distribution and reproduction in any medium or format, as long as you give appropriate credit to the original author(s) and the source, provide a link to the Creative Commons licence, and indicate if changes were made. The images or other third party material in this article are included in the article's Creative Commons licence, unless indicated otherwise in a credit line to the material. If material is not included in the article's Creative Commons licence and your intended use is not permitted by statutory regulation or exceeds the permitted use, you will need to obtain permission directly from the copyright holder. To view a copy of this licence, visit http://creativecommons.org/licenses/by/4.0/.

\section{References}

1. Bensimon G, Lacomblez L, Meininger V (1994) A controlled trial of riluzole in amyotrophic lateral sclerosis. N Engl J Med 330(9):585-591. https://doi.org/10.1056/nejm199403033300901

2. Zoccolella S, Beghi E, Palagano G, Fraddosio A, Guerra V, Samarelli V, Lepore V, Simone IL, Lamberti P, Serlenga L, Logroscino G (2007) Riluzole and amyotrophic lateral sclerosis survival: a population-based study in southern Italy. Eur J Neurol 14(3):262-268. https://doi.org/10.1111/j.1468-1331.2006.01575 $\mathrm{x}$

3. Bensimon G, Doble A (2004) The tolerability of riluzole in the treatment of patients with amyotrophic lateral sclerosis. Expert Opin Drug Saf 3(6):525-534

4. NICE (2001) Guidance on the use of riluzole (Rilutek) for the treatment of motor neurone disease. 20. National Institute for Health and Care Excellence. URL: www.nice.org.uk/guidance/ ta20

5. Leighton D, Newton J, Colville S, Bethell A, Craig G, Cunningham L, Flett M, Fraser D, Hatrick J, Lennox H, Marshall L, McAleer D, McEleney A, Millar K, Silver A, Stephenson L, Stewart S, Storey D, Stott G, Thornton C, Webber C, Gordon H, Melchiorre G, Sherlock L, Beswick E, Buchanan D, Abrahams S, Bateman A, Preston J, Duncan C, Davenport R, Gorrie G, Morrison I, Swingler R, Chandran S, Pal S (2019) Clinical audit research and evaluation of motor neuron disease (CARE-MND): a national electronic platform for prospective, longitudinal monitoring of MND in Scotland. Amyotroph Lateral Scler Front Degener 20(3-4):242-250. https://doi.org/10.1080/21678421.2019.15826 73

6. Brooks BR, Miller RG, Swash M, Munsat TL (2000) El Escorial revisited: revised criteria for the diagnosis of amyotrophic lateral sclerosis. Amyotroph Lateral Scler Motor Neuron Disord 1(5):293-299

7. Morrison KE, Krishna U, Worku DA (2017) Overview and audit against Motor Neurone Disease (MND) Association guidelines of a MND consultant's practice over the last 3 years at the [Hospital]. Res Medica 24(1):65-74. https://doi.org/10.2218/resmedica.v24i1 .1563

8. Centre HaSCI (2014) Use of NICE-appraised medicines in the NHS in England-2012, experimental statistics.

9. Nygren I, Antonova K, Mattsson P, Askmark H (2005) The ALS/ MND prevalence in Sweden estimated by riluzole sales statistics. Acta Neurol Scand 111(3):180-184. https://doi.org/10.111 1/j.1600-0404.2005.00384.x

10. Talman P, Duong T, Vucic S, Mathers S, Venkatesh S, Henderson R, Rowe D, Schultz D, Edis R, Needham M, Macdonnell R, McCombe P, Birks C, Kiernan M (2016) Identification and outcomes of clinical phenotypes in amyotrophic lateral sclerosis/ motor neuron disease: Australian National Motor Neuron Disease observational cohort. BMJ open 6(9):e012054. https://doi. org/10.1136/bmjopen-2016-012054

11. Bellingham MC (2011) A review of the neural mechanisms of action and clinical efficiency of riluzole in treating amyotrophic lateral sclerosis: what have we learned in the last decade? CNS Neurosci Ther 17(1):4-31. https://doi.org/10.111 1/j.1755-5949.2009.00116.x 